\title{
INTERVIEW AVEC PAUL RICOEUR ${ }^{1}$
}

\author{
LE 8 JUILLET, 1991
}

Reagan: Dans les interviews que nous avons faites dans les derniers huit ou dix ans, nous avons discuté vos oeuvres comme Temps et Récit, Du texte à l'action, La Décade à Cérisy, et dans la dernière interview que nous avons faite à Chicago, l'automne dernier, nous avons parlé de Soi-même comme un autre. Pendant cette interview, je voudrais parler un peu plus de votre oeuvre et de votre vie. Par exemple, quand vous étiez jeune, vous avez envisagé une trilogie, une eidétique, une pragmatique, et une poétique de la volonté. En revanche, pensez-vous que vous aviez une archétectonique ou était-il plutôt un voyage intellectuel? Ou, choisissez une autre métaphore.

Ricoeur: Oui, oul. La trilogie que j’annonce dans la préface de la philosophie de la volọnté est nettement inspirée par la trilogie de la philosophie de Karl Jaspers sur laquelle j'avais travaillé pendant les cinq années de captivité avec Mikel Dufrenne entre 1940 et 1945 qui avait abouti, d'ailleurs, dans un ouvrage sur Karl Jaspers. Cette projection correspondait à peu près aux trois parties de la philosophie de Jaspers. La première s'appelait "Exploration du monde;" la seconde,

${ }^{1}$ Cette interview, enregistrée en français à Châtenay-Malabry le 8 juillet, 1991, est la quatrième dans une suite d'interviews avec Paul Ricoeur faites depuis 1982. Ces interviews feront partie d'un livre biographique sur Paul Ricoeur que Charles Reagan est en train de préparer. La transcription est faite par Charles Reagan avec l'aide de sa collègue, Prof. Betty McGraw. Copyright $\odot 1991$ by Charles E. Reagan. 
"L'Existence;" et la troisième, "Transcendence et métaphysique." Et, c'est ainsi que j'anticipais le futur de mon oeuvre.

Mais, j'ai dévié de ce projet par le développement de la philosophie de la volonté elle-même. A savoir que j'avais commencé avec une méthode réflexive empruntée à Husserl avec une certaine inflection existentielle proche de celle de Merleau-Ponty. Mais néanmoins, l'essentiel de l'analyse était réflexive et Husserlienne en ce sens que la réflection atteint non pas des états de conscience contingents ou passagers, mais des structures dominantes de la vie psychique. Telles que, la perception, le projet, l'émotion ce que Husserl appelait l'analyse eidétique.

La rupture de méthode est venue de la rencontre du problème du mal comme une structure fondamentale de la volonté. En un sens, cela pouvait encore apparaître comme le développement du projet initial puisqu'il y a un caractère empirique des formes du mal, qu'il s'agisse de la violence, du mensonge, ou de certaines passions destructrices. Donc, on pouvait dire que c'est la deuxième partie de ce projet. Mais, le changement de méthode imposée, venait des expériences fondamentales tournant autour de ce qu'on pourrait appeler la volonté mauvaise non pas du langage propre et non pas de langage reflexif direct concernant la structure du projet, de l'habitude, de l'émotion, parce que ces expériences ont été structurées autour de l'histoire et dans les grandes cultures, ou autour de récits et de mythes, et sur la base du langage symbolique. Et, c'est donc la symbolique du mal--c'est le titre de ce volume--qui a marqué le tournant, qu'on pourrait dire déjà herméneutique de mon travail.

Alors, cette symbolique du mal, comme je viens de le dire, restait encore partiellement dans le cadre prévu, disons plutôt dans la préface de la philosophie de la volonté. Mais, en fait, la visée contenue avec le projet initial était moins importante que le changement de méthode. Je me suis expliqué beaucoup plus tard, en particulier dans le premier article dans $\mathrm{Du}$ texte à l'action, sur ce que j'appelle la greffe de l'herméneutique sur la phénoménologie. Car, d'une part je maintiens 
la cor:linuité entre phénoménologie herméneutique dans la mesure où l'herméneutique reste fondamentalement une compréhension de soi, et donc, dans une ligne reflexive. Mais, d'autre part les instruments de la compréhension ne sont plus ceux d'une réflexion de caractère transcendental ou eidétique, mais font intervenir la compréhension, l'interprétation, et donc un mode d'intelligibilité autre que celle de la saisie immédiate et intuitive de l'essense des phénomènes mentaux.

Alors, quant à la troisième partie du programme, c'est celui dont la réalisation est la moins évidente. Néanmoins, je prétends que ce que j'appelais déjà une poétique de la volonté se trouve réalisé sous d'autres modalités et j'en citerai précisément trois: d'une part $\mathbf{L a}$ métaphore vive, c'est à dire la créativité du langage au plan de la sémantique, d'autre part, Temps et récit qui est aussi la créativité du langage au plan de la construction des intrigues, et enfin l'investigation de l'imaginaire social sous la forme de l'Idéologie et utopie. En un sens, ces trois ouvrages couvrent une grande partie du champs de la poétique.

Alors, le point où la poétique ne répond pas au programme prévu, c'est évidémment le recours à la transcendence. C'est Jaspers sous une forme sécularisée de la réligion. Or, sur ce point, ma philosophie est strictement agnostique, comme je le dis à la fin de Soimême comme un autre, le phénomène, l'expérience, qui serait le plus près de l'expérience de la transcendence, comme l'expérience de la conscience morale, pẹut être interprétée de façon multiple. La conscience, comme voix intérieure, peut être celle de mes ancêtres, celle de mon être le plus profond comme le voudrait Heidegger, ou la parole d'un Dieu vivant. Mais, la philosophie laisse ouvertes ces possibilités. Et, c'est là que la jonction entre la dimension proprement philosophique et la dimension proprement religieuse intervient.

Mais, je pense que votre seconde question porte sur ce sujet.

Reagan: Ma seconde question est que vous aviez été élévé dans une famille protestante et dévouée et vous êtes resté dans. la foi 
comme croyant et pratiquant, et la question est alors quel est le rapport-s'il y en a un--entre votre foi et votre philosophie?

'Ricoeur: Je tiens beaucoup à l'autonomie de la philosophie et je pense que dans aucun de mes ouvrages philosophiques j'emploie d'arguments empruntés aux domaines des écritures bibliques juives ou chrétiennes. Et, si je le fais, c'est sans argument d'autorité. Je veux dire que, par exemple, je mets sur le même pied la tragédie grecque ou les récits d'Israel, les spéculations métaphysiques néo-platoniciennes ou les interprétations patristiques des écritures bibliques. Par conséquent, il n'y a aucun privilège du religieux en général ou de la tradition judéochrétienne dans l'argumentation philosophique,

Alors, si on dit, "oui, mais si vous n'étiez pas chrétien, si vous ne reconnaissiez pas appartenant au mouvement de l'écriture biblique, vous ne seriez pas intéressé par le problèmé du mal ou, peut-être même, à l'aspect poétique au sens large, l'aspect créatif de la pensée humaine." Alors, à cette objection, je fais toưtes les concessions que l'on veut en disant que personne ne sait d'où viennent les idées qui l'organisent philosophiquement. Nous sommes tous 'visités'--si l'on peut dire--par des projets, des pensées, et personne ne peut rendre compte entièrement de la motivation profonde de ses intérêts spéculatifs. Et, sur ce point, je ne suis pas en défaut, je ne suis pas plus mal placé que quiconque. Personne n'est le maître de l'origine de ses pensées.

Mais, ce dont on est responsable, c'est de ses arguments. Alors, je distingue très nettement, je crois, dans la préface de mon dernier travail entre l'argumentation et la motivation. Certainement, une lecteur serait beaucoup plus sensible que moi je ne le suis à la motivation secrète, religieuse de mon travail. Mais, ce que je soutiens, ce que je prétends avec beaucoup de force, c'est que cette motivation est toujours mise entre parenthèses afin de laisser se former des arguments philosophiques qui sont destinés à tous les êtres rationnels capables de discution comme moi, quelque soit leur position sur le problème religieux. Je ne pense pas, par exemple, que mon traitement ni de la métaphore ni de l'intrigue, et mon interprétation de historiographie--qui 
sont mes derniers travaux--ou mes dernières reflections sur l'altérité emploient des arguments [religieux]. Si l'inspiration ou la motivation intervenait dans le champs de l'argumentation, alors cela fonctionnerait comme un argument d'autorité, c'est-à-dire, le pire des arguments.

La notion d'argument d'autorité est une contradiction dans les termes. Ce qu'il y ait une autorité dans les textes bibliques, alors cela rejaillit d'une prise de position personnelle qui est extrèmement complexe parce qu'on peut dire qu'il y a une sorte de choix mutuel entre être choisi, si l'on peut dire, par un ensemble d'écritures et l'interprétation qui suivent cette écriture, et de les choisir. J'emploie souvent cette formule que c'est un hasard d'être né dans une certaine famille transformé en destin à travers un choix continuel de préférences, pensant que je reçois plus de compréhension de mojmême, des autres, et de l'existence, en général, à partir de l'interprétation qui elle-même peut être très raisonnée d'un fond scriptuaire lié à l'expérience juive et celle des chrétiens de l'église primitive. Alors, je suis bien conscient que cela crée un problème de dualité, mais je préfère les difficultés créées par cette dualité que la confusion qui naîtrait de leur mélange. Je préfère le risque de la schizophrénie à la mauvaise foi du pseudo-argument.

Reagan: Ma prochaine question suit la précédente, c'est-à-dire que votre orientation philosophique et même religieuse qui date des années 30 était chrétienne, pacifiste, socialiste. Mais, je suis frappé par la signification, l'importance, de la guerre dans votre vie personnelle. Par exemple, vous avez perdu votre père au début de la Première Guerre Mondiale; vous avez passé la Deuxième Guerre Mondiale dans un camp de prisonniers de guerre; enfin, vous étiez l'un des leaders des opposants de la politique française pendant la guerre d'Algérie.

Ricoeur: Vous avez bien fait d'évoquer mon premier rapport à la guerre qui est la mort de mon père pendant la Première Guerre Mondiale, parce que ce n'est pas sa perte comme telle qui a été un choc mais le sens qui lui avait été donné dans ma famille. A savoir, 
qu'il était mort pour une juste cause et, bien que mort, il appartenait au corps des vainqueurs.

Or, c'est cette conviction qui s'est effondrée tout d'un coup quand j'avais dix ou douze ans. Sous l'influence de pacifistes chrétiens qui m'ont convaincu que, premièrement, la France avait eu une résponsibilité écrasante dans la déclaration de la Première Guerre Mondiale; et que, deuxièmement, le Traité de Versailles imposé à l'Allemagne était une injustice majeure; et, que troisièmement, cette injustice du Traité de Versailles expliquait largement la montée du Nazisme en Allemagne. Et, cela suscitait pour moi un bouleversement à deux points de vue: d'abord concernant la figure de mon père qui était, dans quelque sorte, détrôné de sa gloire puisque cette figure était rejetée du côté d'une complicité avec l'injustice et la violence de cette guerre. Et, d'un autre point de vue, ce doute concernant le sens de la mort de mon père a donné à mon premier engagement pacifiste un caractère passionnel extrêmement profond que je n'ai jamais surmonté. Parce que toutes les fois que je me suis rendu à la conviction rationnelle que certaines guerres étaient, sinon justes, au moins inévitables, comme la Deuxième Guerre Mondiale, il restait toujours ce doute profond, ce sentiment de l'injustice profonde de la guerre en elle-même. J'ai été toujours partagé entre un côté pacifiste enraciné dans l'émotion et une vue plus rationnelle--même Hegelienne-sur la responsibilité des états et l'emploi nécessaire de la force.

Alors, vous avez fait allusion à la guerre d'Algérie, là j’ai eu la chance d'avoir la possibilité de reconcilier mes deux orientations. Il m'est apparu extrêmement vite que l'indépendance de l'Algérie était une exigence juste. Par conséquent, la guerre que faisait mon pays aux révolutionnaires algériens était une guerre injuste.

Mais je me rappelle des hésitations entre la tendence pacifiste et une certaine rationalité conduisant à justifier la guerre au moment de la guerre d'Espagne. Je viens de revivre ça il y a quelques jours quand j'ai revu les paysages qui pour moi sont inscrits. Ma conscience politique a été largement formée au moment du Front Populaire de 1936 et de 
la guerre d'Espagne en 1937-38. J'étais passionnément du côté des Républicains, c'est-à-dire contre Franco, mais partagé comme mes camarades socialistes de l'époque entre le pacifisme qui leur interdisait d'intervenir et d'autre part la sympathie, la solidarité avec des révolutionnaires espagnols. Or, il se trouve que justement Léon Blum qui était le clúf du gouvernement, partagé lui-même entre ces deux sentiments, a choisi la non-intervention. Et, après-coup on peut se demander si les monstruosités de la Deuxième Guerre Mondiale ne sont pas le résultat de la timidité, du refus d'engagement contre Hitler beaucoup plus tôt.

Mais le côté pacifiste se trouvait être complice d'Hitler. Cela a été pour moi le grand choc de la défaite en 1939. Lorsque je me suis trouvé prisonnier en 1940, je me suis très fortement culpabilisé cette défaite en me disant que mon pacifisme des années précédentes était en grand partie responsable du non-réarmement suffisant de la France en face de l'armement allemand. Par conséyuent, nous avions perdu beaucoup d'énergie à combattre des soi-disant fascistes à Paris, alors que les vrais fascistes étaient à Berlin. Et donc, là c'est un petit peu de mon autocritique politique que je fais. Mais, ça n'empêche que j'ai toujours des bouffées pacifistes.

Ca va peut-être vous amuser, que ce pacificism est la cause même de mon rapport avec les Etats-Unis. Quand je suis revenu de captivité en 1945, j'ai été accueilli dans un petit collège protestant qui était soutenu par des pacifistes Américains, des Quakers. Ce sont eux qui m'ont introduit aux Etats-Unis puisque mon premier poste d'enseignement a été au collège de Haverford qui est un collège Quaker. Si vous voulez, il y a un côté pacifiste Quaker en moi qui de temps en temps trouvait une issue.

Peut-être aussi que mes difficultés à Nanterre comme Doyen s'expliquent par là puisque d'un côté j'ai été pris par des sympathies pour certaines idées gauchistes qui se trouvaient être l'héritage de mon pacifisme de mes douze ans et, d'autre part, un sens très vif de l'institution dont j'étais responsable. Ce que j'appelle souvent mon sens 
Hégélien de l'institution. Voilà pour moi un petit cas de schizophrénie qui m'a plus tourmenté que le précédent entre la philosophie et la religion.

Reagan: Mais, c'était internalisé au début de la Deuxième Guerre Mondiale avant que vous ne soyez prisonnier?

Ricoeur: Vous savez que la guerre était d'une telle brièveté. Oui, vous avez raison de poser la question parce que presque la première fois où j'ai douté de mes choix politiques antérieurs cela a été au moment de la débacle. Lorsque j'étais avec des unités militaires des bretons--très, très courageux--qui étaient encore à l'est de la France, lorsque les allemands étaient déjà à Paris, et de voir fuir tous ces soldats qui abandonnaient leurs : armes, et mêlés à la population, des officiers qui ont vraiment quitté leurs troupes, et de me dire que ça, c'est le produit d'un certain pacifisme...un pacifisme partiellement responsable de cette défaite.

Je sais bien que la défaite ne doit non plus s'expliquer par des sentiments, des émotions, car j'ai vu après ça, en 1945, comme prisonnier, l'effondrement de l'Allemagne à l'est et la même peur des civils et la même décomposition de l'armée. La décomposition d'une armée reflète la décomposition de la volonté politique d'un pays. Alors, je ne veux pas trop m'accabler avec mes responsabilités miniscules mais enfin j'ai vécu cela, au moins j'ai intériorisé la défaite comme un certain jugement moral sur moi-même. Alors, vous pourriez dire que c'est mes idées de culpabilité protestante. Alors, là j'écoute. J'écoute toutes les interprétations.

Reagan: Mais, pendant des semaines, vous étiez un soldat et prêt à faire ce qu'il faut.

Ricoeur: Je n'ai pas de mauvaise conscience comme soldat. C'était une guerre de quinze jours. J'étais fait prisonnier dans des conditions communes, habituelles, à savoir une armée encerclée, réduite à quelques petites unités, quelques poches qui sont réduites les 
unes après les autres, par surnombre, par des attaques aériennes, par des chars. Bon, quand on est perdu dans les champs, dans la campagne, avec une vingtaine d'hommes entourés par des troupes surarmées, ou on est mort ou on est prisonnier. Alors, on peut dire qu'on pourrait choisir la mort. Donc, il y a aucun héroisme d̀ être prisonnier.

Reagan: C'est aussi un sujet pénible pour vous, mais au sommet de votre carrière à la Sorbonne, vous avez choisi de partir pour Nanterre.

Ricoeur: Ah, ce n'est pas un sujet pénible. Non, je suis devenu un spectateur de moi-même là, non, même très à l'aise.

Reagan: C'était avec l'espoir de changer l'université? Parce que, à ce moment-là, vous avez écrit pas mal d'articles sur là ićfórme dans l'université.

Ricoeur: Je crois que je peux dire que j'avais anticipé, pas certes les événéments, mais la configuration de la crise de l'université, qui, à mon sens tenait à trois phénomènes: D'abord, un phénomène démographique; le fait que nous passions d'une université d'élites à une université de masse. Deuxièmement, le caractère inapproprié de l'institution universitaire qui était incapable d'assumer cette mutation étant donné la structure administrative rigide, le système des examens qui consistaient en une série d'épreuves d'élimination. Troisièmement, l'absence de rapport de proximité entre étudiants et professeur. Je me rappelle qu'à la Sorbonne--qui a donc été construite à la fin du siècle dernier--aucune salle n'était prévue pour la réunion des étudiants, même pas des bureaux individuels pour les professeurs. On recevait dans les couloirs, entre deux portes, ou dans la cour de la Sorbonne. Donc, ces masses de plus en plus nombreuses d'étudiants littéralement abondonnés à eux-mêmes sans possibilité de s'adresser personnellement à des professeurs.

C'est pourquoi j'ai choisi d'aller dans cette institution nouvelle avec l'espoir qu'elle aurait beaucoup plus grande autonomie de 
fonctionnement. Et c'est là que je me suis trompé parce qu'elle n'était qu'une projection de la Sorbonne en plus petit. Les défauts de l'institution étaient moins visibles parce qu'il y avait moins d'étudiants, des rapports personnels plus réels, il y avait des bureaux, et choses de ce genre. Mais tout le curriculum des études était exactement le même.

Et du même coup, une institution faible. C'est ce qu'ont très bien compris les gauchistes comme Cohn-Bendit. Et parce que c'était l'institution la plus faible, c'était elle qu'il fallait briser la première. La relation entre Nanterre et Cohn-Bendit n'était pas du tout le hasard. Peut-être c'était assez contingent qu'il se soit trouvé étudiant là; je n'en suis pas sûr. Etant là, il a parfaitement vu que c'était le point sensible. Lui, il était victime d'une illusion symẹtrique de notre propre aveuglement, lui, il se figurait que toutes les institutions d'un pays forment une sorte de chaine comme dans la theorie des dominos (que vous avez eue dans votre politique extérieure...si on perd le Vietnam, on perd...).

Il pensait que si l'institution la plus faíble s'écroule alors, de proche en proche, toutes les institutions s'écroulent. Et, d'une certaine façon, il a failli avoir raison puisque la grande crise de l'université est devenue une crise nationale avec $\mathbf{1 1}$ millions de grévistes dans la rue, et le Général de Gaulle a vacillé; il a failli être emporté par la vague. Et c'est vraiment par sa force de caractère qu'il a réussi à regrouper ses propres troupes. Finalement, l'opinion publique, lassée du désordre, a basculé en sens contraire.

Je crois que l'université française est la seule de toutes les universités dans le monde qui ait été foudroyée par la crise. Ce qui n'a pas été le cas avec Berkeley, ou avec Tokyo, ou avec Berlin. Il n'y a que l'université française qui a été vraiment mise à genoux. En un sens, cela a été une bonne chose, parce qu'on a pu reconstruire un système universitaire beaucoup plus souple. Je prends un exemple: l'Université de Paris, considérée comme une seule université, avec 200 mille étudiants, avec un seul recteur, invisible dans un bureau. Si bien 
que, les décisions à la Sorbonne ou à Nanterre devaient être prises sur place sans qu'on ai l'autorité ou la responsibilité qui appartenait à la tête, au sommet. Des choix, tout à fait absurdes, comme d'appeler la police à la Sorbonne--ces choix-là n'auraient pas été faits s'il y avait eu quelqu'un de responsable plus près, qui aurait eu autorité de prendre d'autres décisions.

Alors, on a reconstruit après ça un système d'autorité beaucoup plus adapté. Il y a treize universités au lieu d'une à Paris. Il y a un président et un conseil d'administration responsables. Les deux seules responsabilités que nous n'avons pas, c'est la responsabilité financière, parce que nous vivons sous le budget de l'état, et, d'autre part, la nomination des professeurs puisqu'elle se fait sur la base d'une commission nationale. Troisièmement, nous devons donner des diplômes de niveau national, puisque les diplômes ne sont pas des diplômes de l'université mais des diplômes nationaux. Donc, le système est resté très centralisé, bien que plus articulé, plus distribué qu'avant la crise de 1968.

Reagan: Mais, vous venez de parler d'appeler la police. Mais c'était la crise à Nanterre deux ans après, n'est-ce-pas, au printemps de 1970 ?

Ricoeur: Plutôt, 1969. J'étais donc l'assistant du Doyen en 1968, et comme j'étais à ses côt's pendant ses décisions, j'étais un peu "en vue". Et, en 1969, lorsque les troubles ont repris, ils avaient un tout autre caractère qu'en 1968. En 1968 il y avait vraiment un projet révolutionaire--absurde, sans doute--mais réel. Cohn-Bendit pensait à un changement profond de la société, des institutions.

En 1969, ce n'est pas du tout le cas. C'était les vaincus de 1968 qui, au fond, avaient le projet d'empêcher l'université de fonctionner. C'est très important de voir cela. Donc, je me suis trouvé en face des gauchistes sans projet, sinon un projet destructeur. Et là, où je retrouve mes dilemmes de tout à l'heure, partagé entre une volonté de 
comprendre, de négocier, et d'autre part, un sentiment de devoir extrêmement fort à l'êgard de cette institution.

Au fond, je crois que j'avais été élu parce que l'assemblée responsable voyait en moi une sorte de personnage double, à la fois capable de négociation et d'autre part de rigueur dans la décision. Et, c'est vrai que cela a marché pendant un an. Je n'ai jamais été protégé dans mon bureau--il a été envahi plusieurs fois. Lorsque les troubles sont devenus dangereux--il y avait des bandes armées de barres de fer qui s'affrontaient entre des ultra-gauchistes maoïstes et, d'autre part, des éléments d'extrême droite. J'ai été littéralement écrasé entre ces deux pressions. Et, comme je refusais d'appeler la police, c'ést l'administration centrale qui me l'a imposé. Un jour, j'ai trouvé la police sur le terrain. J'ai consideré cela comme un échec. J'ai donc empêché la police d'entrer dans les bâtiments, et donc après ça, j’ai démissionné.

Reagan: Mais, René Rémond m'a dit qué ce que vous avez fait c'était de "banaliser" l'université, c'est-à-dire de rendre possible l'entrée de la police. Mais, c'était le Ministre de l'Intérieur qui a envoyé toute une armée de police pour vous embarrasser.

Ricoeur: Oui, je pense que ce vote auquel je n'étais pas favorable résultait de mon conseil affolé. "Banaliser" veut dire que le terrain n'est plus sous la responsabilité de l'université. Vous connaissez Nanterre; c'est assez vaste. On ne peut pas le contrôler. Nous laissons la sécurité et l'ordre aux soins de la police sur les terrains, soyons responsables de l'ordre à l'intérieur des bâtiments. Et, c'était ce partage qui s'est avéré néfaste, parce que la police occupant tout les espaces ouverts, prenait la position d'assiégeant. C'était dangereux pour eux parce que les étudiants, dans les bâtiments, jetaient des machines à écrire, des tables, des chaises, toutes sortes d'objets. Je craignais qu'il $\mathrm{y}$ ait des morts.

René Rémond était, à cette époque-là, mon vice-Doyen et il m’a succédé. Et il a réussi où j'ai échoué. 
Reagan: Mais, il m'a dit qu'il pensait que vous aviez très bien réussi, et si vous aviez tenu le coup pour quelques jours de plus...Il a dit que ce n'était pas lui qui a réussi à mettre fin à la guerre entre les étudiants et la police. C'était "auto-limitant".

Ricoeur: C'est possible.

Reagan: Après avoir démissioné, vurıs avez pris un congé de trois ans de l'université française et vous avez enseigné à Louvain et à Chicago. Pourquoi ces deux universités et comment ces expériences ont-elles changé votre oeuvre?

Ricoeur: Je distingue entre Louvain et Chicago, ce sont deux décisions différentes. J'ai choisi Louvain, d'abord à cause de sa proximité de Paris et donc cela ne posait pas des problèmes de transplantation; et surtout parce que c'est le centre des études phénoménologiques. J'ai pu reprendre ma recherche sur Husserl et le mouvement phénoménologique.

Alors, en ce qui concerne Chicago, mon rapport avec Chicago était antérieur. En fait, je suis allé pour la première fois à Chicago, si je ne me trompe, en 1967 ou 1968, et j'ai reçu un doctorat honoris causa en même temps d'ailleurs que Lévi-Strauss et Raymond Aron. Et, c'est alors que j'ai reçu une invitation d'y venir regulièrement. Donc, mon rapport avec Chicago n'a pas de lien avec la crise de Nanterre. J'avais déjà fait deux semestres à Chicago avant la crise de Nanterre. Il m'a été plus facile après cela de partager mon temps entre la France et les Etats-Unis.

Je pense que celui qui a été instrumental dans le choix de Chicago a été mon ami Mircea Eliade, qui occupait la chaire d' histoire comparée des religions, mais avec lequel j’avais été lié dès 1947 lorsqu'il était à Paris, enseignant à l'Ecole pratique des Hautes Etudes. Donc, c'était une connaissance très ancienne. Le choix de Chicago a été aussi motivé par ceci que je pouvais enseigner à la fois au depart:ment de philosophie, à la "Divinity School", et au "Committee 
on Social Thought," où j'avais été introduit par Hannah Arendt dont j'avais fait la connaissance chez Paul Tillich. Je pense que mon amitié à cette époque-là avec Paul Tillich et Hannah Arendt a joué aussi un grand rôle dans le choix qu'a fait l'Université de Chicago de m'inviter. Nous parlions tout à l'heure de la schizophrénie entre la philosophie et la théologie, alors, j'ai pu vivre institutionellement cette schizophrénie à la "Divinity School" dans une chaire dont le titre est lui-même schizophrénique parce que ça s'appelle "philosophie théologique" ou "théologie philosophique"; je ne me rappelle plus.

Reagan: Et, vous avez suivi Tillich dans cette chaire?

Ricoeur: Oui, c'est ça; j'ai succédé à Tillich dans cette chaire qui s'appelait "John Nuveen Chair."

Reagan: Mais, votre oeuvre a été changée aussi par cette connaissance des philosophes anglais et américains et leurs propres livres?

Ricoeur: Oui, oui je ne sais pas depuis quand date mon intêret pour la philosophie analytique. J'ai enseigné à la Sorbonne dans les années 70's les cours sur Russell, sur Ryle, et après ça, sur Austin et les actes de langage. Alors, mon intérêt dans la philosophie anglaise est relativement ancien. Je pense d'ailleurs que, paradoxalement, c'est Husserl qui m'y a amené parce que Husserl a toujours pris au sérieux ce qu'on appelait à cette époque-là "l'empirisme". J'ai fait des cours d'histoire de la philosophie--à la période de Strasbourg, entre 1948 et 1958--sur Hume et Berkeley. Donc, je pense que mon intêret pour la philosophie anglo-saxonne est très ancien.

Il est devenu plus impératif, si je peux dire, du fait d'être confronté à des étudiants à Chicago qui avaient une formation philosophique purement analytique. Mais, ce n'est que relativement récemment que j'ai intégré la philosophie analytique comme une voie de passage de ma philosophie sur la base de cet argument qui est herméneutique; à savoir, qu'il n'y a pas de connaissance de soi qui soit 
directe, mais qu'elle passe toujours par l'intermédiaire de normes, de symboles. Donc, les analyses objectives, soit de l'action, soit de l'identité personnelle, que je trouvais dans la philosophie analytique nourrissaient pour moi cette réflection indirecte. Je m'explique de cela dans Soimême comme un autre. Le détour par la philosophie analytique est un détour de plus dans une méthode qui repose justement sur des détours. Détour/retour c'est le rhythme de ma respiration philosophique. Mais, j'ai été toujours très sensible à la force d'argumentation de la philosophie analytique qui me paraissait tout à fait en accord avec un très grand soin conceptuel qu'Husserl avait et que les phénoménologues après lui n'ont pas toujours partagé.

Reagan: Mais, après vingt ans d'enseignement à Chicago et des conférences partout aux Etats-Unis et ailleurs, votre oeuvre a été redécouverte en France dans ces dernières années. Par exemple, je peux citer la décade à Cérisy-la-Salle il y a trois ans et très récemment vous avez reçu le prix de philosophie de l'Institut. C'est comme deux carrières en France.

Ricoeur: Je ne suis pas juge, pas l'historien de moi-même. Je n'ai jamais souffert d'une mauvaise reconnaissance et peut-être pour une raison qui tient à ma façon d'écrire parce qu'on pourrait peut-être me reprocher, je n'ai jamais fait attention à mon public. Je veux dire que ma façon de composer mes livres de philosophie répondait à une urgence intérieure, des questions qui s'imposaient que je ne voulais pas fuir. Je me rappelle c'était le premier conseil que j’ai reçu de mon premier professeur de philosophie, lorsqu'il y a un obstacle, vous ne le contournez pas, vous allez droit devant. Alors, j'ai une façon très combative, non pas d'hostilité--au contraire, une sorte de sympathie pour l'adversaire--ce qui fait que j'ai toujours choisi mes vis-à-vis en dehors de ce qui est dans l'avant-scène, les nouveaux philosophes, etc. $\mathrm{Au}$ fond, je m'explique avec mes meilleurs adversaires et c'est plutôt un débat avec moi-même sans tenir compte de la réception de ces livres par le public. 
Je crois que j'avais une bonne réputation comme enseignant, comme professeur. Et au fond, je suis plus satisfait de ma carrière parce que j'ai aidé la formation philosophique de plusieurs générations d'étudiants. Mon rapport avec un public plus large constitue une histoire différente avec une reconnaissance plus tardive et plus complète. Mais, c'est peut-être aussi le bénéfice de l'âge.

Reagan: Enfin, la dernière question. : Pendant la semaine dernière, quand Antenne 2 faisaient de longues interviews, nous avons bien ri quand vous avez raconté comment le Gệnéral de Gaulle disait pendant une interview, "Vous avez manqué de me poser la bonne question qui est celle-ci." Ensuite il se posait une question et puis il y répondait. Alors, je voulais vous donner la chance de faire comme de Gaulle et poser une question.

Ricoeur: Nous pourrions peut-être parlèr des projets d'avenir. C'est une situation toujours très difficile quand on a fini un livre. Il y a un passage qui ressemble, paraît-il, à la dépression qu'on peut avoir après un accouchement. Le problème est plus grave à mon âge. Je dois dire que j'ai trois zones d'intérêt et qui ne se coordonnent pas du tout et je ne sais du tout si ça aboutira à un ouvrage aussi structuré que Temps et récit et Soi-même comme un autre. D'une part, je voudrais continuer mes recherches sur l'idée de justice qui occupe une place importante dans l'éthique qui est au sommet de mon dernier travail. Il se trouve que j'ai été appliqué ici, en France, dans des travaux de recherche d'institution comme l'Ecole Nationale de la Magistrature, sur le problème de la justice, et aussi dans les milieux de prospection économique comme le Commissariat au Plan sur le problème des inégalités et, en particulier, le paradoxe des inégalités justes; i.e. y a-t-il des inégalités justes? Donc, ma discussion ancienne avec Rawls, Habermas, je voudrais la poursuivre sur ce plan-là.

La discussion au point où elle en est actuellement est un peu une impasse entre un universalisme abstrait et procedural à la façon de John Rawls, ou d'une façon différente mais parallèle chez Habermas, et d'autre part, les communautariens, ceux qui se basent sur l'expérience 
historique, concrète. Je suis pris dans cette discussion, ce débat. Je voudrais approfondir les implications de l'idée de justice sous ses aspects formels et ses aspects institutionels, historiques, concrets. Ca, c'est un champ de recherche avec comme annexe une exploration des passions comme structurant l'espace social, l'espace public, à la façon d'Hirschman et d'autres. Avec toujours le même problème, celui de trouver un contenu vivant au règles abstraites de procédures juridiques ou de pragmatique transcendentale à la façon d'Habermas. Ca, c'est tout un ensemble.

Deuxièmement, j'ai repris mes études sur l'historiographie avec deux intérêts. D'une part, comment fonctionner la rationalité dans la reconstruction de l'histoire, et, d'autre part, quel est le statut du passé qui a été mais qui n'est plus, et donc, qui échappe à tous critères de vérification et qui, pourtant, a une sorte de poids de réalité. Alors, je travaille sur une centaine de pages qui doivent être publiées par l'Institut International de Philosophie.

Et puis, alors, le troisième domaine est un essai de recherche de transition entre l'exegèse biblique et la réflection philosophique sur le plan de ce que j'ai appelé plus haut "ma schizophrénie." Car, je maintiens qu'il y a uné intelligence à l'oeuvre dans ces textes de l'exegèse et que la fonction de l'exegèse c'est d'articuler cette intelligence. J'ai toujours soutenu l'idée que le language, le discours, avait une multitude de fonctions. En ce sens, je suis tout à fait du côté du Wittgenstein des Investigations philosophiques, ou les jeux de langage sont d'un monde illimité, et que la philosophie a, entre autres, pour responsabilité de préserver l'autonomie, le bon droit, de ces jeux de langages. Il y a une place pour le langage religieux à côté du langage poétique à côté du langage scientifique.

C'est dans la perspective de ce pluralisme des formes de discours que l'on peut explorer alors des intersections, des interférences d'entre ces sphères. Il y a certainement des rapports entre l'éthique et la réligion, par exemple, au plan de l'agapé, de l'amour. J'ai fait un petit travail sur amour et justice qui anticipe cela. Mais, il y a aussi entre 
l'intelligence historique, vue la place énorme des récits historiques, des intersections avec le discours poétique, avec le lyrisme biblique comme dans le livre de Job. A cet égard, je serais très intéressé à une comparaison entre les psaumes et la tragédie grecque, sur le plan de la plainte, de la lamentation. Nous n'avons pas parlé de ce problème qui me hante de plus en plus, qui a pris la place du problème du mal, de la culpabilité, qui est le mal de la souffrance. Et, 'le problème de l'homme souffrant. Je ne cesse de dire dans mes travaux, que je parle de l'homme agissant et souffrant.

Finalement, les drames personnelles et le spectacle du monde me rendent sensible à ceci, qu'il y a une souffrance imméritée, répandue dans le monde. Et aussi qu'il y a une parole à trouver. C'est le problème d'Auschwitz, le problème de la mémoire et du pardon. Je serais actuellement intéressé à explorer les zones de jonction entre le religieux, et le poétique, l'éthique, l'historique, etc. Je pense que j'ai payé suffisamment pour leur distinction pour avoir le droit de dire quelque chose sur leur conjonction. A mon âge, c'est assez de projets.

Kansas State University

CHARLES E. REAGAN 